\begin{tabular}{|l|l|l|l|l|}
\hline Revista Clío América & ISSN: 1909-941X & Vol. 8 & No. 16 & Julio - Diciembre de 2014 \\
\hline
\end{tabular}

\title{
Empleo y salarios en Argentina en el periodo de la Post-Convertibilidad
}

\author{
Employment and wages in Argentina in the period of Post-Convertibility
}

Nilson Javier Ibagón Martín

Magister en Historia

Universidad Pedagógica

Nacional de Colombia, Colombia

goyobraudel@yahoo.com.ar

Tipología:

Artículo de Reflexión

Fecha de Recibido:

Junio 18 de 2014

Fecha de Aceptación:

Agosto 11 de 2014

Para citar este artículo:

Ibagón, M. N. (2014). Empleo y salarios en argentina en el periodo de la post-convertibilidad, Clío América, 8 (16), 185 - 194
Resumen: El Articulo analiza los procesos de precarización laboral que se han dado en Argentina a la largo de los últimos 25 años, haciendo especial énfasis en el llamado periodo de "post-convertibilidad" o "dólar alto". En este sentido, se estudia para dicho periodo, la existencia de una serie de continuidades económicas y sociales heredadas de la década del noventa, las cuales, ponen de manifiesto una serie de estrategias y políticas empleadas por parte de los sectores económicos dominantes, que les han permitido en buena parte, por un lado, transferir a las trabajadores los costos de las crisis económicas, y por otro, frenar el acceso de estos últimos a una redistribución de la riqueza en tiempos de bonanza y crecimiento.

Palabras claves: Empleo, Salarios bajos, Política Monetaria, Desindustrialización, Política Industrial

JEL: E24, E52, J31, 014, 014.

Abstract: Article analyzes the processes of labor precariousness that have occurred in Argentina during the last 25 years, with particular emphasis in the period of "post-convertibility" or "high dollar". In this direction, the existence of a number of inherited continuities of the nineties is studied. These legacies reveal a series of strategies and policies used by the dominant economic sectors, that have allowed them, on the one hand, transfer the costs of the economic crisis on workers, and secondly, curb the access of this group to a redistribution of wealth in good times and growth.

Keywords: Employment, Low Wage, Monetary Policy, Deindustrialization, Industrial Policy. 


\section{Introducción}

Tras los destrozos económicos y sociales que dejó en Argentina el régimen de convertibilidad ${ }^{1}$, los cuales se vieron condensados en una profunda crisis en la que el desempleo, el subempleo, la pobreza y la miseria alcanzaron dimensiones nunca antes vistas en la historia del país, se establecieron a partir del año 2002 modificaciones importantes en el patrón de crecimiento, que, de acuerdo con algunos sectores políticos concretos, han marcado una distancia considerable con la racionalidad neoliberal de la década del noventa, impulsando con ello una recuperación de indicadores económicos y sociales que han beneficiado y mejorado las condiciones de vida de los sectores populares.

No obstante, al revisar detenidamente elementos económico-sociales trascendentales y sensibles, como son, el empleo y el salario, las visiones optimistas que sostienen la existencia de un cambio económico contundente a favor de los sectores populares durante el llamado periodo de postconvertibilidad, se relativizan y en muchos sentidos pierden validez, ya que, invisibilizan fenómenos que se constituyen en muestra fehaciente de la prolongación de procesos gestados y generalizados a partir de la década del noventa, los cuales han y siguen golpeando a un sector importante de la sociedad Argentina: los trabajadores.

El presente escrito, partiendo de este contexto general, analiza para el periodo de post-convertibilidad la existencia de una serie de continuidades heredadas de la década del noventa alrededor de la precarización laboral en Argentina. Dichas continuidades ponen de manifiesto una serie de estrategias y políticas empleadas por parte de los sectores económicos dominantes, que les ha permitido en buena parte, por un lado, transferir a las trabajadores los costos de las crisis económicas, y por otro, frenar el acceso de estos últimos a una redistribución de la riqueza en tiempos de bonanza y crecimiento.

Para ello, el texto está dividido en dos ejes de

1. Política económica que adoptó el gobierno Argentino en el marco de las reformas estructurales de inicios de la década del noventa del siglo XX. A través de ella se fijo una paridad 1 a 1 entre el peso Argentino y el dólar estadounidense. análisis: el primer eje, con el fin de establecer un punto de partida que permita un ejercicio de comparación entre las políticas de empleo y salarios impulsadas a partir de la adopción del "dólar alto" con las medidas adoptadas en esta materia durante la convertibilidad, se enfoca en la realización de un balance general acerca de los fenómenos de precarización laboral que se instauran a través del modelo neoliberal implantado en Argentina durante la década del noventa, El segundo eje, por su parte, se centra en el análisis de las características generales del trabajo y el salario en Argentina a lo largo de la post-convertibilidad, tarea que nos permite identificar con relación a la década del noventa, tanto, algunos cambios de tipo cuantitativo -aumento del empleo al inicio del periodo-, como, continuidades de carácter cualitativo que evidencian la ausencia de una transformación estructural en las políticas de empleo.

\section{La convertibilidad y sus efectos sobre el empleo y el salario durante la década del noventa del siglo XX}

La política de establecer una tasa de cambio fija, se configuró a inicios de la década del noventa como el mecanismo que le permitiría salir a Argentina de una compleja realidad hiperinflacionaria (Nochteff, 2001). La convertibilidad al dar mayor relevancia al peso propiciaría una estabilización de los precios y consecuentemente frenaría la inflación. Tarea que en el campo práctico, se llevó a cabo con éxito si se tiene en cuenta que en los primeros años de aplicación de la convertibilidad (1991-1994) Argentina experimentó en comparación a los años de hiperinflación un crecimiento económico importante, caracterizado por el aumento del consumo interno, la interrupción de la inflación y la reactivación significativa de la inversión (Basualdo, 2003).

Sin embargo, ligada a ella, se introdujeron una serie de reformas que más allá del control de la inflación, estaban orientadas a beneficiar directamente los intereses de los sectores dominantes. Dichos sectores, luego de una serie de disputas internas se alinearon como bloque a comienzos de los años noventa, promoviendo una serie de presiones políticas tendientes a reducir los efectos nocivos, que según ellos, traía el excesivo intervencionismo del Estado en asuntos 
económicos, el cual, desde su visión particular, introducía obstáculos e interferencias que no dejaban fluir las relaciones de mercado -favorables a sus intereses-.

Siguiendo este principio general, durante el periodo de vigencia de la convertibilidad (1991-2001) se gestaron una serie de políticas económicas que aceleraron de una forma radical la implementación de la racionalidad Neoliberal en Argentina; racionalidad que ya había sido puesta a funcionar desde la Dictadura Militar (1976-1983) y, con la cual, se puso fin al modelo de industrialización por sustitución de importaciones. De esta forma, procesos tales como: la desregulación de la economía, la flexibilización del trabajo, las privatizaciones y desmonte del aparato y control estatal, el endeudamiento externo, y la desindustrialización, caracterizaron una política que discursivamente se proponía, no sólo, como el camino para solucionar los problemas de hiperinflación de finales de la década del ochenta y comienzos de la década del noventa del siglo XX, sino a la vez, como la vía que encaminaría al país a un crecimiento económico que redundaría en el mejoramiento de las condiciones de vida de la población.

No obstante, estas nuevas condiciones económicas en el campo práctico sólo aseguraron la expansión de los beneficios de los sectores dominantes a costa del trabajo y desmejoramiento de la calidad de vida del conjunto de las clases populares, particularmente la trabajadora, quien se vio fuertemente golpeada por los procesos desindustrializadores que se gestaron en Argentina durante la década del noventa. En este contexto, el patrón de acumulación durante el periodo de la convertibilidad, al establecer un giro en el que los sectores financiero y de servicio guiaron la economía por encima de los sectores que se enfocaban en la producción de bienes transables (Basualdo, 2003), tuvo un impacto directo en la reducción del trabajo y el consecuente aumento del desempleo. De acuerdo con Campos, González y Sacavini (2010) en el periodo comprendido entre el año 1991 y el 2001, los sectores no transables se expandieron a una tasa anual acumulada $20,3 \%$ superior a la de la economía en su conjunto, mientras que la producción de bienes creció a una tasa $18,2 \%$ inferior.
Así, las condiciones económicas propiciadas por la convertibilidad, hicieron que los empresarios, por una parte, antes que pensar en inversiones de carácter productivo asociadas a la creación de empleo, se dedicaran en buena parte a la especulación financiera, actividad supremamente rentable para ellos pero con efectos negativos en el conjunto de la sociedad, cuya base dependía del trabajo como fuente de ingresos; y por otra, incentivaran políticas tendientes a la sustitución de mano de obra por bienes de capital. De ahí que se produjera una reducción del espectro productivo, lo que implicó una concentración de la producción industrial en actividades que se sustentaban sobre la base de ventajas comparativas naturales, facilitando así el avance del capital sobre el trabajo.

De esta forma, a lo largo de la década del noventa tanto en momentos de crecimiento económico (etapa dorada de la convertibilidad, 1991-1994) como en momentos de recesión y crisis, algunos de los mecanismos más importantes utilizados por los sectores dominantes para aumentar sus ganancias fueron: la expulsión de trabajadores y la precarización de las condiciones del empleo. Así, a lo largo de la década del noventa se establecen las normas sobre la determinación salarial (se elimina la indexación salarial, se descentraliza la negociación colectiva y se vinculan las variaciones salariales a la evolución de la productividad); se limita el derecho de huelga; se altera el régimen de vacaciones; se privatiza el sistema de prevención de accidentes de trabajo; se ponen en vigencia diversas formas de contratos temporarios que disminuyen el costo para los empresarios (los denominados "contratos basura"); se reducen los aportes patronales a la seguridad social y las asignaciones familiares; disminuyen las indemnizaciones por despido; etcétera. (Basualdo, 2003, p. 57).

Este escenario complejo para los trabajadores argentinos se vio agudizado por la perdida de competitividad de la producción nacional frente a la extranjera. El tipo de cambio impulsado por la convertibilidad hizo que la primera se encareciera frente a la segunda (CENDA, 2010), situación que promovió la perdida considerable de puestos de trabajo tras la quiebra de un número importante de empresas nacionales. En este sentido, a la especu- 
lación financiera se le sumó el aumento desmedido de las importaciones, como factores que introdujeron variables que impactaron negativamente a la industria argentina y consecuentemente a la generación de empleo. En este contexto se produce la quiebra de pequeñas y medianas industrias que estaban enfocadas en el mercado interno. Un caso concreto es el sector manufacturero, el cual, desde las condiciones de competencia introducidas por el modelo económico sucumbe ante las importaciones.

Sumado al incremento de la desocupación y el subempleo durante el periodo de aplicación de la política de convertibilidad (Ver tabla 1), se presentó a la vez un estancamiento y decrecimiento de los salarios. Así, en medio de un contexto donde el desempleo iba en aumento, éste "funcionó como un potente mecanismo disciplinador que afectó la capacidad de negociación de los trabajadores [facilitando] la imposición de la flexibilización laboral y la intensificación de los procesos de trabajo" (Campos, González y Sacavini, 2010, p. 49).

ITabla 1.

Tasas de actividad, empleo, desempleo abierto y subempleo en Argentina 1990-2002. (Total de aglomerados urbanos relevados)

\begin{tabular}{|c|c|c|c|c|}
\hline AÑ & $\begin{array}{c}\text { TASA DE } \\
\text { ACTIVIDAD } \%\end{array}$ & $\begin{array}{c}\text { TASA DE } \\
\text { EMPLE0 } \%\end{array}$ & $\begin{array}{c}\text { TASA DE DESEMPLEO } \\
\text { ABIERTO } \%\end{array}$ & $\begin{array}{c}\text { TASA DE } \\
\text { SUBEMPLO } \%\end{array}$ \\
\hline 1990 & 39,0 & 36,5 & 6,3 & 7,9 \\
\hline 1991 & 39,5 & 37,1 & 6,0 & 8,1 \\
\hline 1992 & 40,2 & 37,4 & 7,0 & 9,3 \\
\hline 1993 & 41,0 & 37,1 & 9,3 & 10,4 \\
\hline 1994 & 40,8 & 35,8 & 12,2 & 12,6 \\
\hline 1995 & 41,4 & 34,5 & 16,6 & 13,6 \\
\hline 1996 & 41,9 & 34,6 & 17,3 & 13,1 \\
\hline 1997 & 42,3 & 36,5 & 13,7 & 13,6 \\
\hline 1998 & 42,1 & 36,9 & 12,4 & 14,3 \\
\hline 1999 & 42,7 & 36,8 & 13,8 & 14,6 \\
\hline 2000 & 42,7 & 36,5 & 14,7 & 16,3 \\
\hline 2001 & 42,2 & 34,5 & 18,3 & 19,9 \\
\hline 2002 & 42,9 & 35,3 & 17,8 & \\
\hline
\end{tabular}

Fuente: Elaboración propia sobre la base de información del Instituto Nacional de Estadística y Censos (INDEC). 
Estas medidas fueron fructíferas para los sectores dominantes al reducir los costes salariales y generar nuevas dinámicas de trabajo, en las que el trabajador sacrificaba beneficios y salvaguardas a cambio de mantener o conseguir empleo. Con ello, las condiciones de vida de la mayor parte de la población Argentina se vieron afectadas, y la pobreza se convirtió en un común denominador. Según Basualdo (2003) este fenómeno se enmarca en lo que él denomina una "Revancha Clasista", a través de la cual "la reducción del salario real promedio, el desempleo, el subempleo, la pobreza y la indigencia registran niveles inéditos" (p.55).

\section{Empleo y salario en la post-convertibilidad: ¿Cambio o continuidad?}

El consenso que habían logrado generar las clases dominantes alrededor de las ventajas de la racionalidad neoliberal se comienza a resquebrajar a partir de 1998, cuando se manifiesta, por un lado, el reavivamiento de conflictos al interior de ellas, y por otro, se da un proceso de reagrupamiento político de los sectores populares (Basualdo, 2003), el cual en 2002, tras cuatro años de recesión, va a ser definitivo en la caída del modelo de convertibilidad. Indicadores como la pobreza, la indigencia, la desocupación y la subocupación alcanzan máximos históricos ubicándose en mayo de 2002 en el 41,4\%, $18 \%, 21,5 \%$ y $18,6 \%$ respectivamente, hecho que dispara el descontento social y simultáneamente enfrenta a los sectores dominantes alrededor de qué fracción asumiría los remanentes de los costos de la crisis, que ya no podían ser -como comúnmente se solía hacer- trasladados a los sectores populares (Azpiazu y Shorr, 2010).

De ahí que con la Crisis desatada entre los años 1998 y 2001, se desarrollan una serie de disputas entre los sectores dominantes, -que hasta ese momento se habían reagrupado en un bloque para impulsar las políticas neoliberales-, en torno del tipo de cambio que, según ellos, se necesitaba para salir de la debacle económica. Dicha disputa vinculó principalmente al "empresariado productivo", el cual aunque había visto crecer sus negocios y operaciones, se había rezagado en comparación a los sectores financieros y de servicios, y a estos últimos, quienes en el periodo de la convertibilidad habían crecido enormemente. Dentro de estas dinámicas de confrontación, el empresariado productivo se ve perjudicado, configurando un esquema de "ganadores" y "perdedores" (Shorr y Wainer, 2005), en el que el "perder" implica seguir ganando desde indicadores no tan desarrollados y extendidos como el del "ganador". En otras palabras, al interior de los sectores dominantes no hay pérdidas considerables, ya que los cambios de política económica en el caso de Argentina a lo largo de casi cuarenta años han permitido establecer mecanismos de salvaguarda de sus intereses.

Sin embargo, para los sectores financieros y de servicios y, el empresariado productivo, la convertibilidad que hasta ese momento había sido "su caballo de batalla", agotó su potencial de generación de riqueza, condición que exigía - para ellos- un cambio en la política económica. En este sentido, y nuevamente al igual que a inicios de la década del noventa, las sectores dominantes, luego de una disputa alrededor de qué facciones pasarían a ejercer la hegemonía y cuáles quedarían relegadas a posiciones de relativa subordinación dentro del bloque dominante -proceso en el que salen victoriosos los sectores productivos- (Azpiazu y Shorr, 2010), logran establecer un nuevo consenso desde discursos que planteaban la búsqueda del mejoramiento de la economía nacional y con ello la optimización de indicadores como la producción, el trabajo y el ingreso, ya no, alrededor de un tipo de cambio fijo como en aquel momento, sino ahora, en torno de una "salida devaluacionista", que curiosamente estos mismos sectores habían rechazado tajantemente años atrás. Es de aclarar que, este cambio de posición de los sectores dominantes alrededor del tipo de cambio, no obedece a una preocupación legitima por parte de éstos alrededor del deterioro de las condiciones de vida de la mayoría de la población, sino por el contrario, de acuerdo con Shorr y Wainer (2005) se explica por el agotamiento de la convertibilidad, como política que aseguraba la defensa de los intereses económicos de los sectores dominantes. En este sentido, cuando la convertibilidad dejo de ser rentable y funcional para dichos sectores, éstos comenzaron a posicionar discursivamente la necesidad de modificarla.

De esta forma, con el triunfo de la política devaluacionista y la implementación de lo que se denomina 
modelo del <<dólar alto >>, el sector financiero que hasta ese momento había orientado el crecimiento de la economía cede su lugar privilegiado a los sectores "productivos"; cambio que teóricamente desde la visión particular de sectores académicos, políticos y económicos iba a impactar positivamente en indicadores socio-económicos duramente golpeados en el periodo de la convertibilidad como lo fueron, el trabajo y el salario. No obstante, y pese a las cambios económicos introducidos a través de la política de "dólar alto", la precariedad del empleo y los bajos niveles salariales, se constituyen en continuidades que se extienden desde la década del noventa hasta la actualidad, sin que hayan podido ser resueltas satisfactoriamente (CENDA, 2010), principalmente debido a que el desarrollo productivo, particularmente el industrial, se ligó directamente al tipo de cambio, y no, a una política de renovación y reestructuración del perfil productivo. Por ejemplo, a partir del abandono de la convertibilidad, el sector exportador, el cual supuestamente se iba a convertir en el motor de transformaciones positivas en el trabajo y el salario de los argentinos, ha producido el efecto contrario anclando gran parte de sus ganancias y expansión, en la caída efectiva del salario y la precarización de las condiciones laborales. En este sentido, se puede afirmar que durante la posconvertibilidad no se ha superado la regresiva distribución del ingreso, la persistencia de altos niveles de desocupación y precarización laboral, y la perdida del poder adquisitivo del salario (Shorr y Wainer, 2005),

Complementariamente, los fenómenos de concentración económica y extranjerización -legados del periodo de convertibilidad- antes que ser rebatidos, se han seguido consolidando, hecho que ha facilitado el mantenimiento de serios problemas en la generación de empleo y la mejora de los salarios, ya que, la industria determinada por la extranjerización, por una parte, genera pocos puestos de trabajo al estar sustentada fundamentalmente en capitalintensivo, y por otra, asegura parte de sus ganancias en el mantenimiento o profundización de salarios bajos. En este sentido, "a medida que aumenta la extranjerización de la economía, se profundiza un tipo de especialización productiva que consolida la ausencia de un cambio estructural" (Shorr, 2013, p. 57), hecho que facilita el mantenimiento de la precariedad laboral y salarial.

Así, desde la vigencia de la post-convertibilidad aunque Argentina mejoró indicadores económicos asociados a la recomposición del ciclo de acumulación, no ha logrado un avance real en la inclusión económica y social de los sectores trabajadores (Shorr y Wainer, 2005). Esta no inclusión, se ve reflejada particularmente, en el mantenimiento de un modelo productivo que asegura que gran parte de las ganancias de los sectores dominantes, estén soportadas en el deterioro de los salarios reales y la precarización laboral. De ahí que se pueda afirmar que en la posconvertibilidad "existe un cambio claro en el patrón de crecimiento, pero no hay un nuevo modelo de acumulación" (Shorr, 2013, p. 47) que permita posicionar como motores de crecimiento y redistribución del ingreso tanto al trabajo como al salario.

De acuerdo con Shorr y Wainer (2005), tempranamente en el periodo de la posconvertibilidad se generan una serie de procesos económicos y sociales asociados al salario y el trabajo que, explican en parte, el por qué en Argentina en medio de un contexto de recuperación y crecimiento económico, mientras una proporción reducida de la población se beneficiaba de las políticas económicas, las condiciones de vida de la gran mayoría se seguían deteriorando. Entre estos procesos se destaca

a) La erosión de ingresos que trajo aparejada la inflación; b) la vigencia de un "ejército de reserva" de grandes dimensiones (a pesar de la caída que se ha verificado en la tasa de desocupación); c) las modalidades que asumió la creación reciente de empleo (fuerte dinamismo del trabajo no registrado y salarios relativamente reducidos para los nuevos empleos "en blanco"-en muchos casos por debajo de los valores que determinan la línea de pobreza- d)el hecho que los aumentos salariales fijados por decreto recayeron casi exclusivamente sobre los trabajadores en blanco. (Shorr y Wainer, 2005, p. 64).

Estos indicadores, contrastan con la que tal vez es la mayor diferencia que se puede establecer en materia de trabajo entre la convertibilidad y la primera etapa de la post-convertibilidad: la generación de empleo. 
En dicha etapa la disminución de la tasa de desempleo, marca una diferencia considerable con la tradición que se había generado en la década del noventa del siglo XX alrededor de una economía que expulsaba trabajadores (Ver Gráfico 1).

\section{G Gráfico 1.}

Tasa de Desempleo en Argentina 2003 - 2012. (En tasas anuales medias)

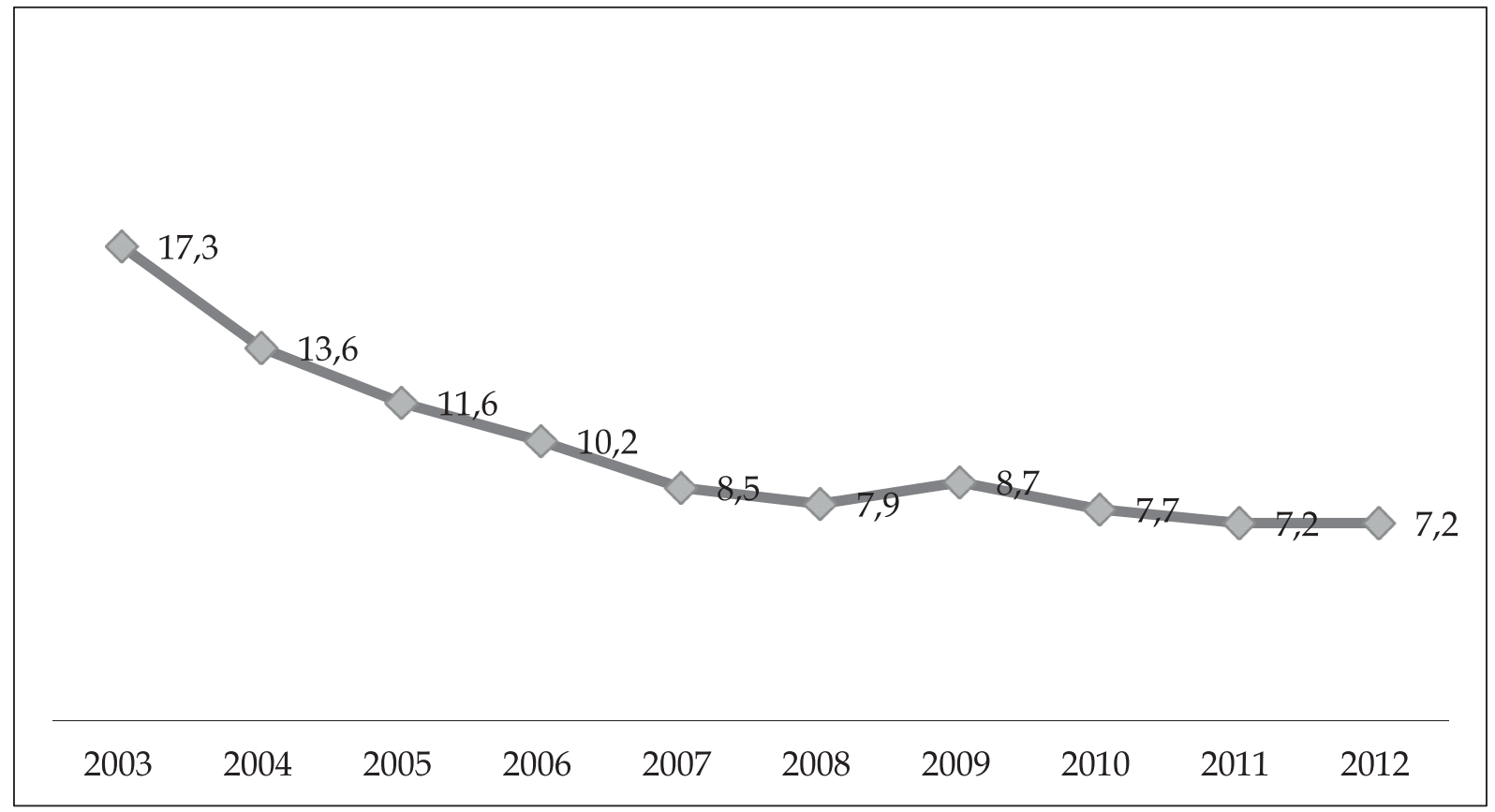

Fuente: Elaboración propia sobre la base de información de la CEPAL

Durante los años 2003 a 2007, en los que Argentina tuvo una tasa de crecimiento promedio anual acumulativo de su PIB del 8,5\% -el cual contrasta con un crecimiento negativo del $4,2 \%$ entre los años 1999 al 2002-, se dio un aumento significativo en la generación de nuevos puestos de trabajo, hecho que se tradujo en la disminución de la tasa de desempleo, la cual "pasó de niveles cercanos al $20 \%$ en el primer trimestre de 2003 a valores inferiores al $10 \%$ en $2007 "$ (CENDA, 2010, p. 25).

Aunque, desde una mirada desprevenida, este aumento de puestos de trabajo y la disminución del desempleo pueden ser entendidos como un éxito rotundo de las transformaciones económicas introducidas en la posconvertibilidad, al ser evaluados detenidamente y a profundidad, encubren una serie de fallas estructurales que al no ser resueltas reprodujeron -y en muchos casos agudizaron- en la práctica y en la vida cotidiana de los trabajadores las condiciones laborales y salariales degradadas propiciadas por el modelo económico de la década del noventa. Por ejemplo, el poder adquisitivo de los salarios no tuvo una recuperación real que le permitiera igualar valores históricos anteriores, así como tampoco se redujo considerablemente el empleo no registrado -para el año 2010 seguía afectando al $35 \%$ de los asalariados-, a su vez no se produjo un mantenimiento sostenido en la generación de empleo -el cual se redujo a partir del año 2008- (CENDA, 2010); carencias que se dieron principalmente por la ausencia de una política real de industrialización. 
En otras palabras, "los avances" en materia de trabajo y salarios durante la recuperación económica que experimentó Argentina en el periodo comprendido entre 2003 y 2007 luego de la crisis (1998-2002) no beneficiaron realmente a los trabajadores, quienes, aunque crecieron en número, no lograron recuperarse de la devastación que soportaron en la crisis de finales del siglo XX quedando expuestos nuevamente ante los embates de la desaceleración de la economía que comenzó a presentarse a partir del año 2008, y que nuevamente los expuso directamente al desempleo y la sub-ocupación. En esta medida, aunque "la nueva macroeconomía modificó las condiciones en que el sistema industrial [creció] (...) no provocó un cambio estructural [en éste]" (Fernández y Porta, 2007, p. 64), manteniendo por ejemplo, lógicas empresariales que ubican al salario como un gasto que hay que reducir, hecho que impidió la consolidación de la calidad del empleo conforme aumentaba la cantidad.

De esta forma, aunque en la posconvertibilidad se da una importante creación de puestos de trabajo y consecuentemente se mejoran algunos indicadores sociales y dinámicas laborales, entre las que se pueden destacar: la recuperación de la negociación salarial, la implementación de políticas tendientes a reducir la no registración en ciertos sectores, la ley de movilidad jubilatoria y las moratorias provisionales, la extensión del sistema de asignaciones familiares a través de la asignación universal por hijo, etc. (Barrera, Fernández, Manzanelli, 2013), la ausencia de una verdadera transformación en el perfil industrial de Argentina, y por lo tanto, la falta de un cambio significativo en la forma en la que este país se inserta en la división internacional del trabajo, relativizó dicho éxito. El tipo y condiciones de trabajo que se generaron no permitieron superar temas como la precariedad e informalidad en éste, ni mucho menos la reducción constante de los salarios reales que se vieron presionados hacia abajo por un contexto inflacionario grave. Mientras tanto, y en contraste con la dura realidad de los sectores asalariados, el modelo económico impulsado desde la post-convertibilidad facilitó "una concentración económica global a favor de la elite empresarial" (Azpiazu, Manzanelli y Shorr, 2011, p. 103), quien en gran medida aumentó sus ingresos y consolidó un crecimiento sostenido a costa del mantenimiento y agudización (en algunos casos) de las precarias condiciones laborales y salariales que desde la implementación del modelo neoliberal han afectado a la mayoría de la población.

Por otro lado, se debe tener en cuenta, que la mejora de los indicadores de generación de empleo a partir del año 2008 comienza a detenerse; año en el cual "salen a la luz las contradicciones e insuficiencias de una política basada en el manejo del tipo de cambio como único instrumento para lograr una expansión sostenida de la producción, el empleo y los salarios reales" (Campos, González y Sacavini, 2010, p. 70). En este sentido, el desempleo vuelve a ubicarse como un fenómeno que altera las dinámicas de la vida cotidiana de los argentinos, reactivándose por parte de los empleadores prácticas típicas de la década del noventa entre las cuales se destacan los despidos generalizados, la no renovación de contratos, la intensificación del trabajo y la reducción de turnos y salarios, acciones a través de las cuales buscaban recuperar la rentabilidad obtenida en el periodo 2004-2007 (Azpiazu y Shorr, 2010). Sin embargo, es necesario resaltar que a diferencia del periodo de convertibilidad, el escenario de desempleo iniciado a partir del 2008 no alcanzó las dimensiones dantescas de la crisis de finales de siglo XX, debido principalmente a la intervención del Estado y la puesta en marcha de algunas políticas paliativas que redujeron su impacto en la población, las cuales se han mantenido hasta la actualidad. Entre dichas políticas asistenciales se destacan: Plan integral para la promoción del empleo y los programas de inserción laboral; Programa Jefes y Jefas de Hogar Desocupados (PJJHD); Plan Nacional de Seguridad Alimentaria; Plan Familias por la inclusión social; Plan Nacional de desarrollo Local y Economía Social "Manos a la obra".

No obstante, dichas políticas no logran ocultar ni las fuertes transferencias de ingresos desde el trabajo hacia el capital, que se siguen presentando (Shorr, 2012), ni tampoco se han constituido como una salida que viabilice el mejoramiento de las condiciones laborales, ya que, " (...) siguen reconociendo en la carencia de empleo la principal raíz de la pobreza, desconociendo la todavía persistente precariedad y 
vulnerabilidad de los empleos existentes, aún de los que se están creando" (Rodríguez y Reyes, 2006, p. 59). Dicho desconocimiento facilita, por ejemplo, que en la actualidad desde una plataforma política que se presenta así misma como defensora de lo social, se pongan en marcha medidas que en medio de la búsqueda de frenar problemas como la inflación, golpean duramente a los sectores populares a través de la reducción significativa del salario real vía devaluación. Hecho que en el fondo es una muestra clara que en Argentina se ha vuelto una constante el que en tiempos de crisis los "sacrificados" siempre sean los sectores populares, mientras que las elites económicas mantienen sus privilegios dándose incluso el lujo de crecer.

\section{Discusión y Conclusión}

Teniendo en cuenta los elementos de análisis hasta aquí expuestos, se puede afirmar que, en Argentina a lo largo del periodo de post-convertibilidad pese a las modificaciones que se han dado en materia del patrón de crecimiento económico, no se ha gestado un cambio estructural en las políticas de empleo, situación que ha impedido el mejoramiento efectivo de las condiciones de vida de la mayoría de la población, más allá, de la instauración y fortalecimiento de ciertos programas sociales de corte asistencialista. En otras palabras, los avances que se han dado en materia económica luego de la crisis de comienzos del siglo XXI, se han relativizado y en buena parte perdido, en tanto que, los sectores dominantes han seguido propiciando la precarización laboral como mecanismo que les asegura, en buena parte, la defensa, mantenimiento y ampliación de sus beneficios económicos particulares. De ahí que, tanto la informalidad, la reducción de los salarios reales y la falta de "calidad del empleo", sigan siendo variables vigentes en un contexto político en el que paradójicamente se habla de mejora efectiva de la calidad de vida de la población.

Un aspecto fundamental que explica esta realidad, es la no transformación real de la producción en medio de un proceso de crecimiento económico importante como el experimentado por Argentina luego de la crisis de comienzos del siglo XXI. Dicha ausencia, a la larga, ha facilitado que problemas económicos que se presentaron durante la convertibilidad y fueron piezas claves de la crisis económica a finales del siglo XX -desindustrialización, crisis laboral y redistribución regresiva del ingreso- sigan manteniendo su vigencia de forma matizada en un contexto económico que supuestamente despliega estrategias para superarlos. Estrategias que se caracterizan por estar enmarcadas en medidas de corto plazo, que alivian momentáneamente las tensiones, pero que a la postre no solucionan los problemas estructurales que agobian desde hace un tiempo considerable a la sociedad Argentina, especialmente a las sectores trabajadores.

En este sentido, el caso argentino es la evidencia fehaciente de los destrozos que genera en una economía en recuperación, la falta de una política seria y contundente que asegure, por un lado, la generación de empleo y, complementariamente, el fortalecimiento de su calidad, entendidos como criterios que permiten en buena parte una redistribución efectiva de la riqueza y consecuentemente la optimización de las condiciones de vida de la población. Así pues, la experiencia económica Argentina de debacle-recuperación-crisis que ha caracterizado su historia reciente, brinda pistas valiosas a los países latinoamericanos sobre los limites reales que tiene el crecimiento económico cuando éste no es acompañado de políticas macroeconómicas que aseguren, no sólo, su duración en el tiempo, sino a la vez, su cualificación en términos de generación de desarrollo.

\section{Referencias Bibliográficas}

Azpiazu, D., Manzanelli, P. y Schorr, M. (2011). Concentración y extranjerización en la economía argentina en la posconvertibilidad (2002- 2008). Cuadernos del CENDES (76), 97-119.

Azpiazu, D. y Shorr, M. (2010). La difícil reversión de los legados del neoliberalismo. La recuperación industrial Argentina en la posconvertibilidad. Revista Nueva Sociedad (225), 31-47.

Barrera, M., Fernández, A. y Manzanelli, P. (2013). Trabajo y pobreza: virtudes y desafíos de la posconvertibilidad. Revista Autogestión XXI. Debates urgentes para otra economía, (2), 5-17. 
Basualdo, E. (2003). La reformas estructurales y el Plan de Convertibilidad durante la década de los noventa. El auge y la crisis de la valorización financiera. Revista Realidad Económica, (200), 42-91.

Fernández, C. y Porta, F. (2007). El crecimiento reciente de la industria argentina. Nuevo régimen sin cambio estructural. En: Kosacoff, B. (ed.). Crisis, recuperación y nuevos dilemas. La economía argentina 2002-2007. (pp. 63-107). Buenos Aires: CEPAL.

Campos, L., González, M. y Sacavini, M. (2010). Convertibilidad y después. El mercado de trabajo en los distintos patrones de crecimiento. Revista Realidad Económica (253), 48-81.

CENDA. (2010). La anatomía del nuevo patrón de crecimiento y la encrucijada actual. La economía argentina en el período 2002-2010. Buenos Aires: Atuel.

Nochteff, H. (2001). La experiencia argentina de los 90 desde el enfoque de la competitividad sistémica. Buenos Aires:
FLACS0-Argentina, Recuperado de http//bibliotecavirtual. clacso.org.ar/ar/libros/argentina/flacso/no_8_NOCHTEFF _Experiencia_Argentina.pdf

Rodríguez, C. y Reyes, M. (2006). La política social en la Argentina post-convertibilidad: políticas asistenciales como respuesta a los problemas de empleo. Buenos Aires: CIEPP.

Schorr, M. (2013). Nuevo Patrón sobre un viejo modelo: el problema de la concentración y la extranjerización en la economía argentina. Revista Debate Público, (5), 47-64. (2012). Argentina: ¿nuevo modelo $0<<$ viento de cola>>? Una caracterización en clave comparativa. Revista Nueva Sociedad, (237), 114- 127.

Shorr, M, y Wainer, A. (2005). Argentina: ¿Muerte y resurrección? Notas sobre la relación entre economía y política en la transición del <<modelo de los noventa > al del <<dólar alto>>. Revista Realidad Económica, (211), 32-65. 